\title{
LA UROLOGÍA PREILUSTRADA. DE LA UROLOGÍA DEL REAL COLEGIO DE CÁDIZ A LA DE LA VENERANDA TERTULIA
}

Manuel Romero Tenorio, Domingo Solano Castro, Mํo José Ledó Cepero, Reyes Romero de Soto, Oscar Valencia Vergara, Amalia García Vázquez, Dolores Romero de Soto, Juan Soto Villalba y José Álvarez Ossorio.

Servicio de Urología. Hospital Universitario Puerta del Mar Cádiz. Cádiz. España.

\begin{abstract}
Resumen.- El estudio de la actividad urológica del occidente andaluz se encuentra contenido en las "Observaciones" impresas y manuscritas de las Memorias de la Regia Sociedad de Medicina Sevillana y en aquellas pertenecientes al Real Colegio de Cirugía de Cádiz. Comprende un período que abarca desde 1693 hasta el primer tercio del siglo XVIII. Se consolida la Veneranda Tertulia, Felipe V concede "Cédula Real"; es el 1ㅇe Octubre de 1701. Inicia su andadura Médico - Quirúrgica la "Regia Sociedad de Medicina y demás Ciencias de Sevilla". Hay que recordar que toda la cirugía andaluza y especialmente la de Cádiz la través del Real Colegio de Cirugía) estuvo presente en la Regia Sociedad. Ordoñez de la Barrera, Sánchez
\end{abstract}

Bernal, Fray Ambrosio de Guibeville, Juan Lacombe, Pedro Virgili y otros muchos fueron fundacionales de este extraordinario acontecimiento.

La formación quirúrgica y, por ende, la urológica tenía su raíz y fundamento en los anfiteatros anatómicos, (Sevilla 1731) y (Cádiz 1728) definidos "impíos" por la Iglesia.

José Celestino Mutis (1750 - 53) y Pedro Fernández Castilla (1741) aíslan a la Universidad de este nuevo movimiento.

Hubo una intensa relación entre Cirujanos de la Armada y la Regia Sociedad, siendo socios desde su fundación: Guibeville (1719); Sánchez Bernal (1719); Gregorio Arias (1729); Gaspar de Pellicer (1729); Lacombe (1730); Fernández Castilla (1741); Calero (1789). Siendo el principal protagonista Luis Montero, verdadero paradigma con proyección hacia la centuria siguiente, de influencia netamente francesa junto con Ramos, ambos alumnos de Beaumond, anatómico de reconocido prestigio.

Palabras clave: Historia de la Medicina. Preilustración. Occidente Andaluz. Historia de la Urología.

Summary.- The study of the urological activity in the Andalusian occident is contained in the printed and handwritten "Observations" of the records of the Royal Society of Medicine of Seville and the Royal College of Surgery of Cadiz. They encompass the period from 1693 to the first third of the 18th century. By the first of October 1701, the "Veneranda" gathering consolidates and Philip V gives the "royal warrant". The "Royal Society of Medicine and Other Sciences of Seville" starts its medical-surgical path. It should be reminded that all 
the Andalusian surgery and specially the one from Cadiz (through the Royal College of Surgeons) was present in the Royal Society. Ordoñez de la Barrera, Sánchez Bernal, Fray Ambrosio de Guibeville, Juan Lacombe, Pedro Virgili and many others were founders of this extraordinary event.

Surgical training and, by extension, urological training had their root and basement in the anatomical amphitheaters (Seville 1731 and Cadiz 1728), which were considered ungodly by the Church.

José Celestino Mutis (1750-53) and Pedro Fernandez Castilla (1741) excluded the university from this new movement.

There was and intense relationship between Navy surgeons and the Royal Society, being members since its foundation: Guibeville (1719); Sánchez Bernal (1719); Gregorio Arias (1729); Gaspar de Pellicer (1729); Lacombe (1730); Fernández Castilla (1741); Calero (1789). The main protagonist was Luis Montero, real paradigm with projection to the next century, having a neat French influence altogether with Ramos, both of them being Beaumond's alumni / an anatomist of recognized prestige).

Keywords: History of Medicine. Pre illustration period. Western Andalucia. History of Urology.

\section{INTRODUCCIÓN}

Ya en el ocaso de 1697 agonizaba la centuria del saber urológico heredado del Renacimiento; conocimientos estancados sin originalidad tras la vivencia de un ejercicio profesional afanoso y poco innovador de cirujanos del XVII conformes con lo establecido. Pérez Albacete destaca entre el evidente tedio urológico las figuras de Andrés León, de Martín Arredondo, Viana, López de León y algún otro, responsables más de transcribir experiencias ajenas que de aportar experiencia propia de valor quirúrgico o anatómico (24).

Paulatinamente la reacción no se hizo esperar, poco a poco los inquietos se corporativizan para luchar contra el inmovilismo doctrinal establecido universitario, rebelándose al poder de los claustros, dogmáticos, pétreos, llevando su ilusión a verdaderos doctos menospreciados por falsos doctores, contra los que tendrán que luchar las Academias y Colegios de Cirugía del Occidente Andaluz.

Fruto de este corporativismo de progreso, nace un trozo señorial de la cultura Sevillana en 1697, la Veneranda Tertulia, en 1700, la Regia So- ciedad de Medicina y demás Ciencias de Sevilla, que se transformaría en 1830 en la Real Academia de Medicina Hispalense. Años después, el Colegio de Cirugía de Cádiz, crisol de excelentes cirujanos ilustrados.

Así se hizo factible una intensa relación de conocimientos y experiencias entre cirujanos andaluces con afinidad específica urológica, especialmente evidente entre el Colegio de Cádiz y la Regia Sevillana.

Pero todo tiene su fin. Es un hecho histórico de especial trascendencia el decreto de Carlomarde, ministro de Fernando VII, anulando la totalidad de las Academias de Medicina de España y que fueron reconstituidas como Reales Academias de Distrito, con estatutos de igual rasero a todas las del Reino en 1831 (25). Real Orden de 31 de Agosto de 1830. Reglamento General para el régimen literario e interior de las Reales Academias de Medicina y Cirugía del Reino, formado por la Junta Superior Gubernativa de estas dos partes de la ciencia de curar.

Así quedaba mermada la actividad quirúrgica en general y la urológica en particular de este florido período que con espíritu renovador aparecía en 1700 con Carlos II, que aprobó las "Constituciones" y Felipe V, que en 1701 concede "Cédula Real", transformando lo que en un principio fue "Veneranda Tertulia Hispalense Médica-Chímica, Anatómica y Matemática" y que aparece en la aprobación de Ordóñez de la Barrera (lámina III) a la obra de Melero Examen Pacífico (1) que encontramos en los archivos de la Real Academia de Medicina y Cirugía Hispalense.

Pero no menos importancia tenía la labor que paralelamente se desarrollaba en Cádiz de la mano de los cirujanos navales del Real Colegio de Cirugía de la Armada con la misma ideología y propósito.

Al final ambas corporaciones sufrieron el mismo trato $(4,5)$.

Pero ¿Cuáles fueron las bases de esta afinidad de criterios constatada en Andalucía Occidental durante este largo y prolífico período llustrado? ¿Cuáles fueron sus autores comunes?

Se basó fundamentalmente en la intensa relación existente entre los cirujanos con actividad urológica que nacieron con la "Veneranda Tertulia" (1697) y aquellos pertenecientes a la Sociedad Médico-Quirúrgica de Cádiz, cirujanos de la Armada fundadores del Colegio de Cirugía un tercio de siglo después. 
Toda esta actividad fue descrita por Francisco Javier Laso de la Vega, primer historiador de la Sociedad Médico Quirúrgica de Cádiz y posteriormente analizada por Orozco en $1981(31,8,5)$.

En segundo lugar, la unificación en Cádiz de la enseñanza Médico-Quirúrgica.

Francisco Canivell, cirujano naval y socio cirujano de honor de Cádiz, en 1791 consigue oficialmente este reconocimiento, el Colegio pasa a ser Real Colegio de Medicina y Cirugía mediante el "Reglamento aprobado y mandado observar por el Rey Nuestro Señor para el régimen científico.... Y para el gobierno de los profesores que ejerzan esta parte de la Ciencia de curar en todo el Reino (1827). José Celestino Mutis estudiaba como cirujano naval en Cádiz, trasladándose a Sevilla en 1750, es el principal referente en este momento de la unión entre Cádiz, Sevilla y Granada $(11,10,26,20)$.

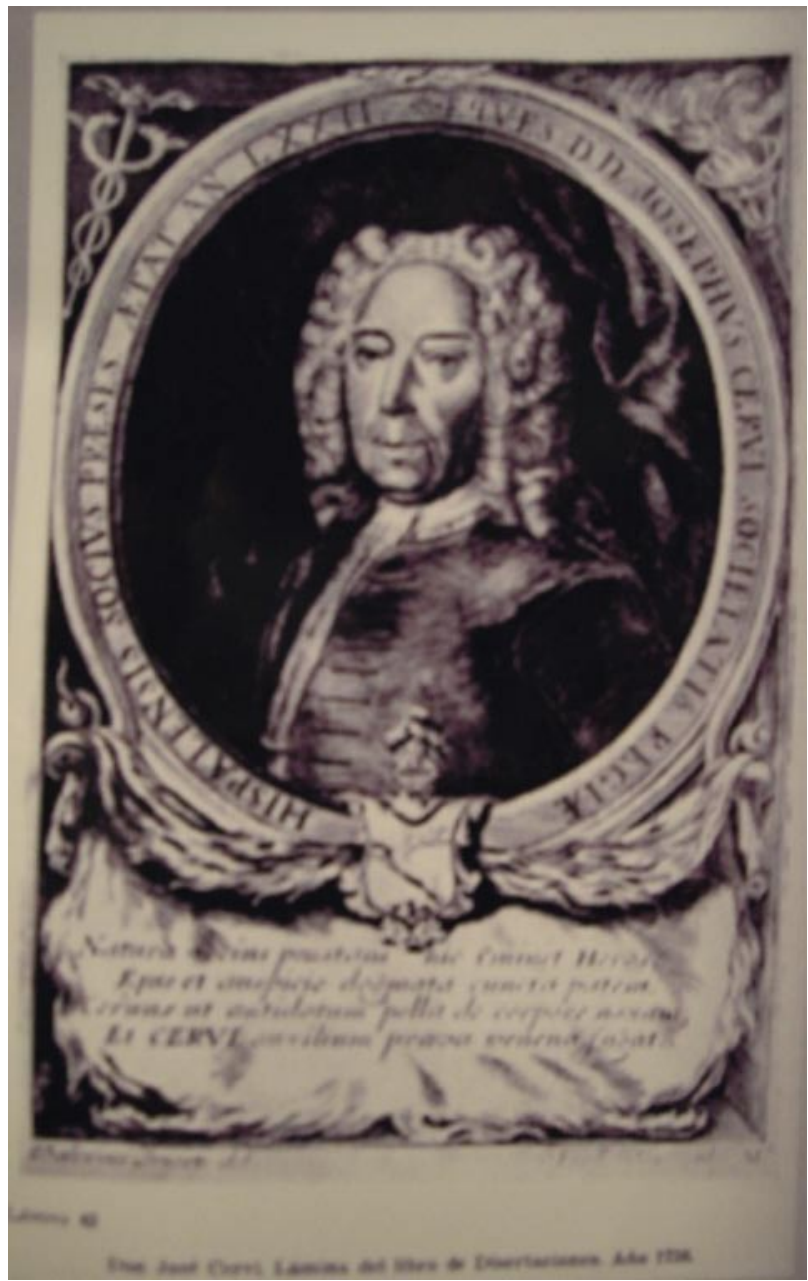

FIGURA 1. D. José Cervi. Presidente y Protomédico de S.M. 1748.
En tercer lugar la expansión de muchos de estos cirujanos a otros hospitales de Andalucía Occidental.

Es necesario en este punto resaltar las magistrales opiniones vertidas por Gregorio Marañón en la Revista de Occidente (1935) y Vida e Historia (1941) y de Antonio Orozco en sus estudios sobre el Real Colegio de Cirugía Gaditano. Plasmados en 33 artículos sobre el Real Colegio de Cirugía de la Armada la labor de los cirujanos navales y su relación con otras corporaciones (29).

La especialización en cirugía no estaba determinada pero sí la inclinación de muchos de estos cirujanos a expresar su experiencia en determinadas actividades urológicas.

Alrededor del entorno creado por Muñoz y Peralta comienza la fluida comunicación de Sevilla y Cádiz. Juan Ordóñez de la Barrera, cirujano de Cámara y Gentilhombre de Artillería del Real Presidio de Cádiz (12), Sánchez Bernal, Protomédico de la Armada (13), y el director del Hospital de esta última, Fray Ambrosio de Guibeville (14), con sus sucesores en el cargo Juan Lacombe (32) y Virgili (22), comienzan una sosegada revolución basados en:

- la defensa a ultranza innovadora sobre el dogmatismo universitario.

- la defensa del poder fáctico, pero no equivocado de la lglesia (27).

Esto último tuvo como consecuencia un logro administrativo sin parangón y desgraciadamente no extensible al resto de Andalucía y de España, como antes se ha mencionado, la creación de una "jurisdicción exenta" que en 1791 unifica los títulos de Médico y Cirujano. La historia lo reconoce en Francisco Canivell. El Real Colegio de Cádiz se transforma en el Real Colegio de Medicina y Cirugía, de extraordinario interés urológico (21).

Consecuencia de ello fue la promoción en Barcelona (1760) y Madrid (1787) de unos Reales Colegios con alumnos de Cádiz y Sevilla pero sin el mismo apoyo administrativo (28).

Se planteó para este logro dos necesidades:

- la enseñanza que debería fluir de sus anfiteatros anatómicos. Lacombe (1728) Virgili (1730)

- el condicionamiento ético que suponía tener cirujanos preparados para poder embarcarlos y atender médica y quirúrgicamente a la marinería (23). 
Todas estas circunstancias históricas se plasmaron en intercambios personales y de conocimientos de interés urológico:

Fray Ambrosio de Guebeville: aunque Cirujano Mayor y Director del Hospital de Cádiz, ingresó en la Academia Sevillana en 1719. Es referido en Disertaciones urológicas, pero no se le conoce ninguna aportación personal (15).

Juan Sánchez Bernal, Protomédico de la Armada, Académico de la Sevillana desde 1719 (noticias de su actividad en prólogos de disertaciones, sin aportación personal) (6).

Gregorio Arias León, cirujano de la Real Armada, ingresó en 1729 en la RAMS. Se le conocen cinco disertaciones académicas sólo en la manuscrita "sobre hernias", 1755 aparece un profundo conocimiento sobre la anatomía testicular. Fundamentos técnicos, quelotomía, separación de arteria ilíaca, fajas y ligaduras... pasaron los tiempos de curar las hernias mediante castración (7).

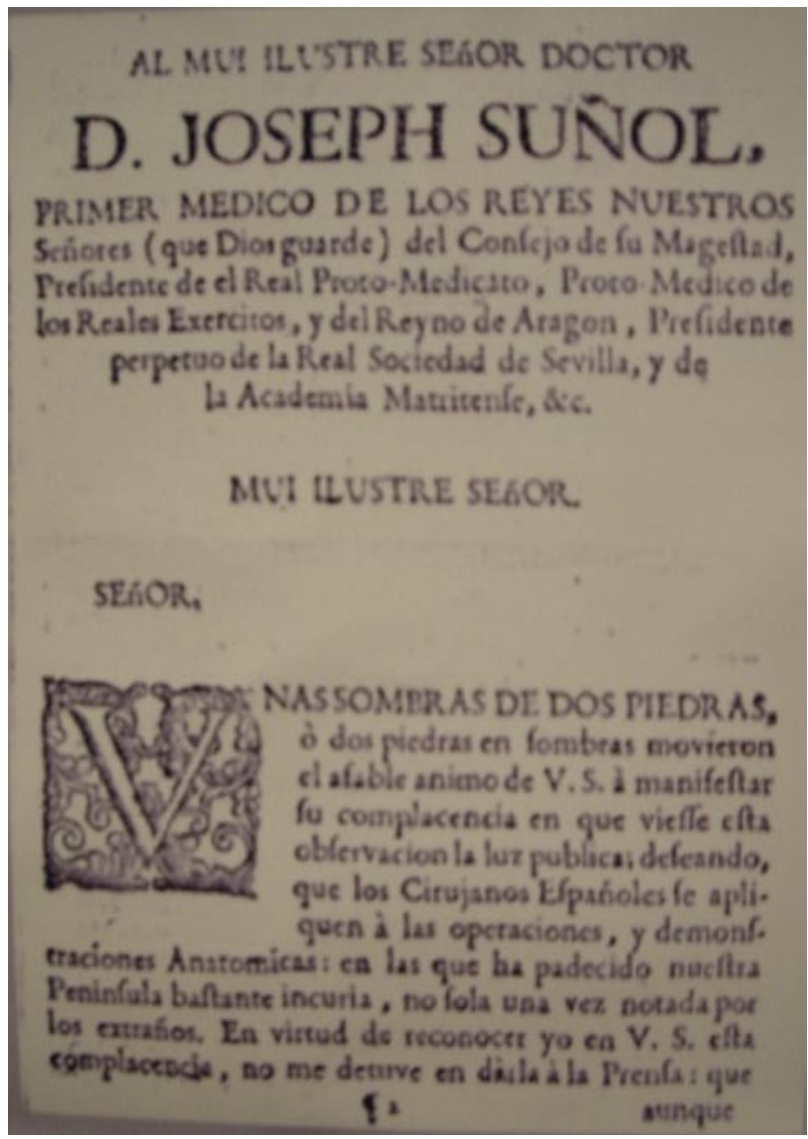

FIGURA 2. Preámbulo de Luis Montero dirigido a Suñol en "Operación lithothomica y demostración anatómica". Archivo Central Universidad de Sevilla.
Gaspar de Pellicer, miembro del Hospital de Marina en 1728 y de la RAMS 1729 (referido sólo en prólogos). No se le conoce aportación urológica personal pero describe la anatomía del aparato genito - urinario. Fue fundamentalmente anatómico y maestro del Real Colegio $(33,9)$.

Leandro de la Vega, Médico del Puerto de Santa María y Protomédico de la Armada, miembro de la RAMS (1737) realiza consideraciones sobre la orina en la "censura crítico - médico epistolar" (1745). En su obra Pharmacopea de la Armada, publicada en 1760, luce su relación con Sevilla "Regiae Scientarum Hispalensis Acadeniae socii". En ella se destacan emolientes para tratar los procesos irritativos vesicales (2).

Bartolomé Francisco Calero y Torres, nacido aproximadamente en 1704, ya que en 1784 refería tener 80 años de edad. No consta entre los alumnos del Real Colegio de Cádiz; debió ingresar en la Armada antes de su creación. Su relación con la medicina Sevillana es importante. Memorias (1765) Tomo I y

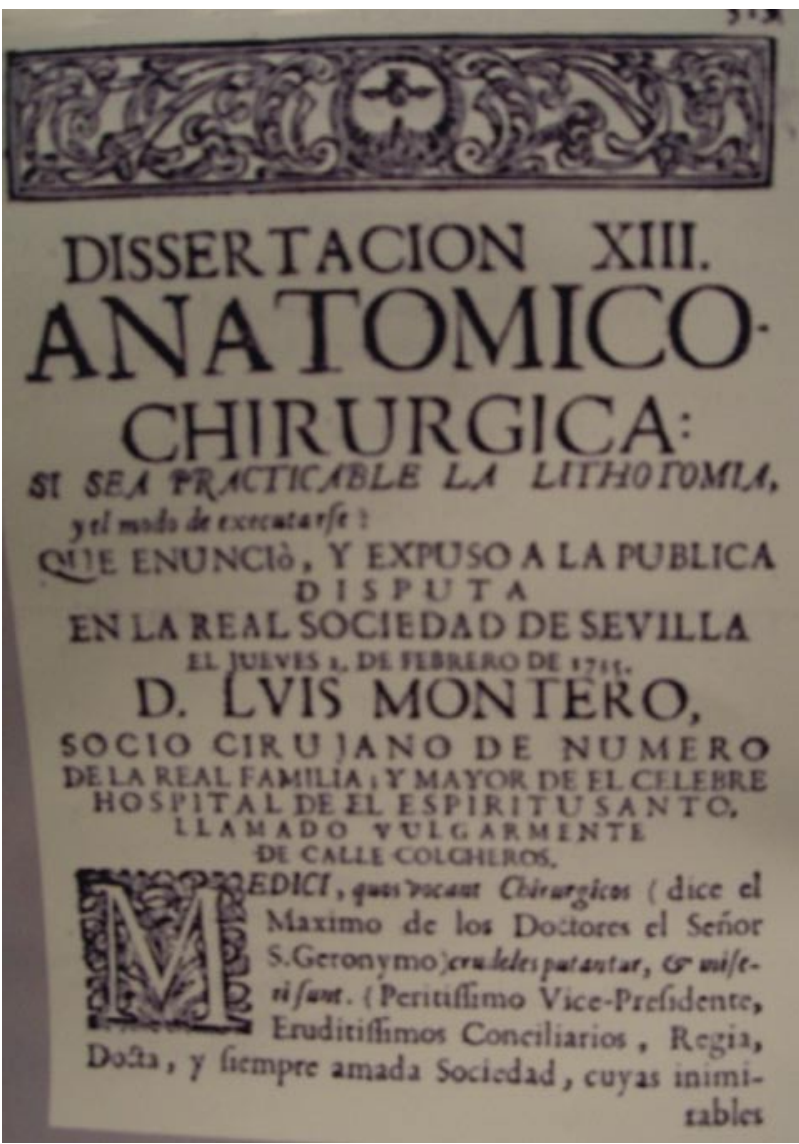

FIGURA 3. Disertación de Luis Montero. Portada de observación lithothomica. Hospital del Espíritu Santo, 1748 - BUS. 
IV. En el Archivo de la Academia Sevillana se conservan veinticuatro disertaciones (1753 - 1774). En la observación 696 y 697 descubre la etiología, diagnóstico y tratamiento del hidrocele "hernia acuosa entre tegumentos del escroto y la primera membrana propia de los testes, anasarca del escroto" Aplicaba de primera instancia tratamiento tópico, si no era eficaz recomendaba la punción y como último extremo la castración.

En la observación no 701 habla de la "carúncula" llamándola "papiloma de veru montanum" indicando dilataciones progresivas. Indudablemente describe el tratamiento de cateterización uretral en prostáticos exclusivamente $(16,18,19)$.

Diego Rodríguez del Pino, natural de Villa de Aguilar (Córdoba) ingresó en el Real Colegio de Cádiz en 1760. Disertó sobre la "Historia de la anatomía de los Riñones, Uréteres y Vejiga y el mecanismo con que se hace la secreción de la orina y análisis de este líquido" posteriormente "demostración anatómica de los riñones, uréteres y vejiga" obteniendo la plaza de Catedrático de Anatomía de la Regia Sociedad. Su salud es precaria por lo que insta al Intendente de Marina su retirada. Esto conlleva el no obtener el título de cirujano. En 1767 entra en prisión por ejercer la cirugía sin título (denuncia del Tribunal del Protomedicato de Sevilla).

Rodríguez del Pino regulariza su situación y ejerce en Córdoba como Cirujano Mayor del Hospital Cardenal. Se traslada a Madrid (1783) como catedrático de San Carlos.

En el Real Colegio de San Carlos presenta veinticinco observaciones a las Juntas Literarias y una sobre los "diversos métodos de operar el hidrocele" en 1803 (3).

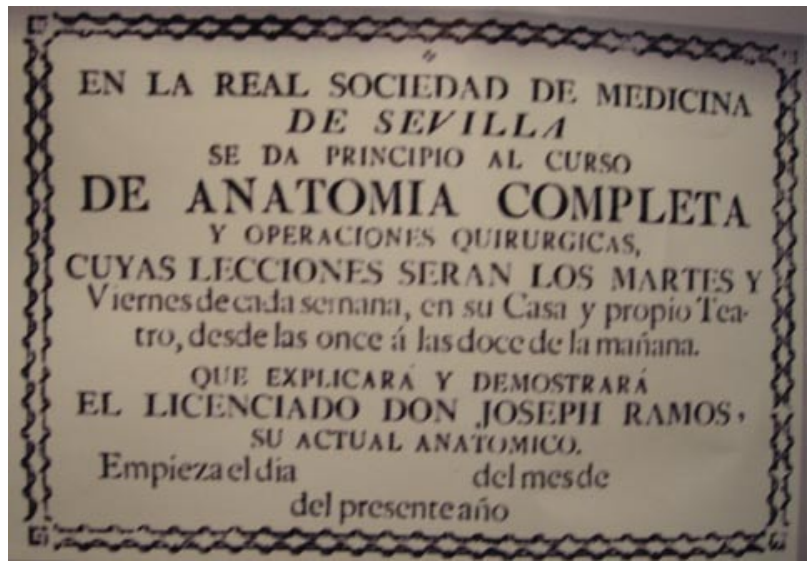

FIGURA 4. Portada de las convocatorias oficiales sobre cursos de anatomía. Ramos Carrasquilla, 1741.
Pedro Balmaña, catalán, natural de Mataró, Colegial de Marina (1751) y en relación con la RAMS desde 1765. La observación de una congestión purulenta en el hipocondrio derecho sugiere la descripción de pionefrosis (17).

José Ramos Carrasquilla fue uno de los anatómicos que mayor renombre alcanzó en la Sociedad Hispalense. Cordobés, natural de Baena, ingresó en el Real Colegio de Cádiz ya bastante mayor, con 21 años de edad, excelente estudiante, pero al realizar su embarque como segundo cirujano en 1782 en el navío Santísima Trinidad no es apto para la navegación y aunque estaba propuesto para cirujano primero se constata que es inhábil para la Armada. La Regia Sociedad de Sevilla encarga a Canivell que proporcione un buen anatómico y no duda en proponer a Ramos que había sido disector anatómico, tomando posesión el 19 de Junio de 1789.

Demostrada la valía quirúrgica de Ramos y la precaria situación económica de su sueldo se le concede derecho a vivienda en la propia casa de la Sociedad.

De José Ramos conocemos numerosas aportaciones pero específicamente las urológicas quedaron manuscritas.

La numerada como 698: "Del hidrocele y de sus especies, manifestando cual sea el método más sencillo y seguro para lograr su curación radical" (30 de Abril de 1788) establece en su escrito un tratamiento mediante punción y drenaje permanente con hebras de hilo blanco aplicando suspensorio compresivo.

Era dominador de la vía hipogástrica como derivación urinaria, vía no recomendada en esta época; lo explica detalladamente el 3 de Marzo de 1796 "De una observación de un caso de retención de orina particular y de sus consecuencias" (4).

Merecen mención aparte aquellos cirujanos que no tuvieron relación directa con el Colegio Gaditano y la Armada pero que supusieron trasiego de información y experiencia práctica en toda Andalucía.

Gómez de Espinosa (disertación $n^{\circ} 750$ ) y Luis Montero (disertación $n^{\circ}$ 699) sobre la circuncisión utilizando sonda acanalada y la sección a tijera.

Francisco Pizarra (disertación no 689) y Francisco Gómez (disertación n 690) en el tratamiento del síndrome irritativo provocado por la infección urinaria. 
Las técnicas de evacuación urinaria surgen paralelas técnicamente a aquellas litotómicas pues a menudo las vías de punción en cateterismos no exitosos eran similares. Varias disertaciones describen su protocolo. José Manuel Jiménez (disertación nํ 691), Francisco Rodríguez (disertación no 692), Miguel Ruiz Tornero (disertación $n^{\circ} 693$ y 695), José Ramos (disertación n 694 y 698), Bartolomé Calero y Torres (disertación $n^{\circ} 696$ y 697 ).

La litotomía en cuanto a técnica tuvo relevancia en Juan de Herrera (disertación no 704 y 705) que seguía los principios de Heister y el principal ejecutor y protagonista Luis Montero de influencia francesa y basada en las descripciones anatómicas de Martín Martínez.

Montero publicó "Observación de operación litotómica y demostración anatómica, operada y demostrada en el insigne Hospital del Espíritu Santo" (Sevilla 1748). Luis Montero ejerció en el Hospital del Espíritu Santo, vulgarmente "el de la calle Colcheros". Cirujano Mayor de dicho Hospital y del Hospital del Amor de Dios, en donde coincidía con Blas Beaumond y Ramos Carrasquilla, siendo el equipo quirúrgico más acreditado de toda esta centuria, en la vertiente urológica, constatamos que las relaciones entre Cádiz y Sevilla fueron permanentes y fructíferas (30).

Hemos descrito las personas y hechos más sobresalientes en el entorno médico y específicamente urológico de la íntima corriente existente entre las dos entidades médico - quirúrgicas de relevancia en toda España del siglo XVIII, la Academia Sevillana y el Real Colegio de Cirugía de Cádiz y su influencia sobre todos los Hospitales de Andalucía Occidental (Córdoba, Osuna, Granada, etc.)

La urología supo conservar el tesoro heredado del Renacimiento y renovar lo que el barroco médico durmió, espíritu renovador semblanza de la llustración.

\section{BIBLIOGRAFIA y LECTURAS RECOMENDADAS ( ${ }^{*}$ lectura de interés $y^{* *}$ lectura fundamental)}

1. Antecedente de la "Regia Sociedad de Medicina y demás Ciencias" de 1700 fue la Veneranda Tertulia Hispalense Médico - Chimica Anathomica y Matemathica de 1697. Cf. Sánchez de la Huerta, Gabriel (1970): Prólogo a Hermosilla Molina, A.: Cien años de Medicina Sevillana. Sevilla.
2. CABRERA ALFONSO, J.R.: "Discurso de apertura del curso Académico de la Real Academia de Medicina y Cirugía de Cádiz”. 191, 1999.

3. CABRERA ALFONSO, J.R.: "Evolución histórica de la Escuela Anatómica Gaditana (1748 - 1844)". Tesis Doctoral. Cádiz 327, 1985.

4. CABRERA ALFONSO, J.R.: op. cit. (su segundo apellido y aspectos de su biografía se deben a este autor) pp. 344-348.

5. CLAVIJO, S.: "Historia del Cuerpo de Sanidad de la Armada". San Fernando, 725, 1925.

6. CLAVIJO, S.: op. cit. P.726.

7. En: Disertaciones Médicas. Sevilla, 1736, Disertación $n^{\circ} 463$.

8. OROZCO AQUAVIVA, A.: "El modelo de enseñanza en el Real Colegio de Cirugía de Cádiz en el siglo XVIII". Gades, 18: 87, 1988.

9. FERRER, D.: "Historia del Real Colegio de Cirugía de Cádiz". Cádiz, 345, 1961.

10. GARCÍA PÉREZ, J.: “Aportación a la vida y obra de Pedro Castelló”. Univ. Salamanca, 1981.

11. HERMOSILlA MOLINA, A.: "Cien años de Medicina Sevillana". Sevilla, Edit. Excma. Dip. Prov. Sev. 710, 1970.

Para su biografía cf. Ameller, Carlos Francisco (1798). Elogio póstumo de Francisco Canivell y de Vila. Cádiz; Plata y Marcos, M. de la (1864): Estudios biográficos, bibliográficos de la Medicina Militar Española. Madrid, pp.171-182; Alivio Lambea, M. P. (1980): Biografía de Don Francisco Canivell y Vila. Tesis Doctoral. Barcelona. Univ. Autónoma.

12. HERMOSILLA MOLINA, A.: "Historia del Cuerpo de Sanidad de la Armada". op. cit., p. 721 - 725. Vid. También Clavijo, Salvador, San Fernando, 1925.

13. HERMOSILLA MOLINA, A.: op. cit., p. 716. Ofrece noticias sobre él Ferrer, Diego (1961) Historia del Real Colegio de Cirugía de Cádiz. Cádiz. pp. $99-100$.

14. HERMOSILLA MOLINA, A.: op. cit., p. 711. El francés Jean Le Combe y Pandrino (1680 - 1748) era natural de la Villa de La Guardia provincia de Le Morgues (Francia). Profesor Cabrera Alfonso: Sobre su relación con la Armada cf. fundamentalmente: Clavijo, Salvador: El primer Cirujano Mayor de la Armada Don Juan Lacombe, en el $2^{\circ}$ Centenario de su muerte. Revista General de marina, 1949. pp. $559-571$.

15. HERMOSILLA MOLINA, A.: op. cit., (13).

16. HERMOSILLA MOLINA, A.: "Cien años de Medicina Sevillana". Legajo, 96, 1784.

17. HERMOSILLA MOLINA, A.: 1970 op. cit. pp. 671 y ss.

18. HERNÁNDEZ MOREJÓN, A.: "Historia Bibliográfica de la Medicina Española”. 7: 298. 1850.

19. Las Comunicaciones Urológicas de Bartolomé 
Calero son: 696, 697, 701. Del hidrocele o hernia acuosa (1758); Sobre el hidrocele y su radical curación. Cf. Hermosilla Molina, A. (1970), op. cit. Pp. $677-687$.

20. Libro de Reales Ordenes y Decretos, Arch. Fac. Med. Cádiz. pp. 1 y 2.

21. Libro de Reales Ordenes y Decretos, Arch. Fac. Med. Cádiz. pp. 1 y 2.

22. OROZCO AQUAVIVA, A.: "Aportación a la Medicina Española de los Hospitales de Marina en Cádiz”. Aula Militar de Cultura. Cádiz, 49, 19961997.

23. OROZCO AQUAVIVA, A.: op. cit. pp. 50 - 69.

24. PÉREZ ALBACETE, M.: "La Urología en el Renacimiento y el Barroco". Historia Biográfica y Bibliográfica de la Urología Española, Madrid, Edit. Edicomplat., 43, 2000.

25. R.O. 31 de Agosto de 1830. Reglamento General para el régimen literario e interior de las Reales Academias de Medicina y Cirugía del Reino. Madrid. 1831.

26. Reglamento aprobado... y para el gobierno de los profesores que ejerzan esta parte de la Ciencia de curar en todo el Reino. Madrid. 1827.

27. RIERA, J.: "Cirugía Española Ilustrada y su comunicación con Europa". Valladolid, Univ. de Valladolid, 1976.

28. ROMERO TENORIO, M.: "La Litotomía en la Urología Española del siglo XIX (1800 - 1899)". T. Doc. Univ. Cádiz, 1986.

29. ROMERO TENORIO, M.: op. cit. (28).

30. ROMERO TENORIO, M.: op. cit. (28).

31. Sobre el origen de esta Sociedad. cf.: Orozco Aquaviva, Antonio: Francisco Javier Laso de La Vega historiador de la Sociedad Médico - Quirúrgica de Cádiz. An. Real Acad. Med. Y Cir. Cádiz, XVIII (1981). Extraordinario.

32. Sobre la rivalidad del Protomédico Sánchez Bernal con el Cirujano Mayor Lacombe, v.: Clavijo, S. op. cit. (1925), pp. 99 - 100. Sobre la del Protomédico Diego Porcell con Virgili, v.: Ferrer, Diego. Op. cit. (1961). P. 96.

33. Varias disertaciones médico - teórico - prácticas, anatomo - quirúrgicas y chimico - farmaceúticas, Sevilla 1736, pp. $341-343$. 